\title{
THE MORBID ANATOMY OF INFANTILE MALNUTRITION IN CAPE TOWN
}

\author{
BY \\ J. A. H. CAMPBELL \\ From the Department of Pathology, University of Cape Town
}

(RECEIVED FOR PUBLICATION APRIL 9, 1956)

In recent years several detailed descriptions have been published of the morbid anatomical findings in malnourished children. Waterlow (1948) reported fatty liver disease from the West Indies and suggested that the children died from hepatic failure, while Hill (1951), working in the same area, described the condition of serous hepatosis. Davies in discussing kwashiorkor in Uganda (Trowell, Davies and Dean, 1954) felt that atrophy of the pancreas and other enzyme-secreting glands was the most important aspect of the condition, while the type of malnutrition seen in Johannesburg is still regarded as infantile pellagra by Gillman (Gillman and Gillman, 1951; Gillman and Gilbert, 1954). As a result of these papers one has an impression of several somewhat distinct pathological forms of malnutrition occurring in different parts of the world. These apparent distinctions, however, largely reflect differences of terminology and interpretation rather than of pathological findings, which, with the exception of those described in serous hepatosis, present a fairly constant pattern of retarded growth, fatty liver, pancreatic atrophy and skin lesions. The findings to be presented in this paper deviate only in their detail from this general pattern.

Apart from these regional differences, various clinical forms of malnutrition have been recognized and include kwashiorkor or its local equivalent, simple nutritional oedema, marasmus, paedatrophy and others. Although a clinical distinction between kwashiorkor and marasmus is made in Cape Town, chiefly on the absence of oedema in marasmus, a preliminary study of the material to be reported here showed that pathological distinction between these conditions was not feasible and in this paper they will be regarded as essentially similar expressions of the single entity of malnutrition.

No attempt will be made to cover the vast literature dealing with infantile malnutrition: this has been done by the authors already cited and by the many others included in their bibliographies.

\section{Material and Methods}

Post-mortem material was studied from $\mathbf{4 0}$ coloured and native children who suffered clinically from malnutrition. Adequate control material was available and was examined with particular care in six cases. Malnourished children suffering from any form of tuberculosis were excluded from the series.

For the purposes of this paper a coloured person is one of mixed blood with native, Hottentot and European as the chief elements, and a native is a member of one of a number of pure tribes native to southern Africa. Among the $\mathbf{4 0}$ malnourished children were 10 Natives, 30 Coloureds ( 23 males and 17 females) and the ages ranged from 4 to 30 months with an average age of 13 months.

All material was fixed in formol-saline for 24 to 48 hours ; frozen sections were stained with Sudan IV in Herxheimer's solution and paraffin sections with haematoxylin and eosin, van Gieson's stain, by silver impregnation methods for reticulum fibres and for the Prussian blue reaction with free iron.

\section{Morbid Anatomical Findings}

The great majority of children were in a state of poor general nutrition when they came to necropsy. There was an obvious loss of subcutaneous fat and a decrease in muscle mass that varied from moderate degrees of wasting to extreme emaciation. There were, however, a few exceptions as four of the children were well nourished, a fifth with gross oedema was described as not obviously wasted, and in one other child the fat depots in the limbs were well filled though adipose tissue was absent from the abdominal wall.

The detection of oedema at necropsy was sometimes hampered by chilling of subcutaneous tissues during the body's refrigeration, and even in fatal cases oedema present on the child's admission to hospital sometimes disappeared during treatment. For what the post-mortem observations are worth, however, oedema was recorded in 15 children. Ten children showed serous effusions and two of them, both peritoneal, were considerable in amount. 
Twenty-two children showed some form of skin rash. There was a good deal of variation in the extent of the lesion in different children; its form varied and there was no typical distribution, virtually any part of the body being affected. The changes were not confined to the exposed areas of the body nor were they even more severe there. When the lesion was well developed it showed as a a brown to blackish hyperpigmentation with fine or coarse scaling, which with desquamation led to depigmentation. Ulceration occurred and was commonest on the pinnae of the ears and around the perineum. Purpura was present - in addition to a typical skin rash in four children and was recorded in the absence of other skin changes in two children. Convincing evidence of a haemorrhagic tendency was not found elsewhere in the body in these children with purpura. Angular stomatitis was recorded in 10 children and moniliasis of the upper respiratory and upper gastrointestinal tracts in three. Microscopy of the scaling pigmented lesions showed parakeratosis, some degree of hyperkeratosis and thinning of the Malpighian layer with atrophy of hair follicles and sebaceous glands. Pigmentation was due to melanin which was found both in basal and upper layers of the epidermis and abundantly in the hyperkeratotic and parakeratotic layers.

A fatty liver has been a constant feature in descriptions of infantile malnutrition except for the report by Walters and Waterlow (1954) from West Africa. In this series some degree of fatty change was seen microscopically in 37 of the $\mathbf{4 0}$ children. The colour of the liver at necropsy varied from pale yellow to bright orange, the deeper shades being presumably partly contributed by bile, for one has noticed that many fatty livers from cases of malnutrition develop a green mottling after 24 hours fixation in formalin. The most severely fatty livers floated in water and those slightly less affected floated in formol-saline. 'Floating livers' were present in 13 children. Microscopically, the severest grades of fatty change showed every cell or practically every cell in the liver lobule greatly distended by a large fat globule, so that the section resembled adipose tissue. This severe form of fatty change was present in 23 children. At the other extreme were those livers where only a minority of cells in a lobule were completely occupied by a single large fat droplet, and the degree of change was modest or slight. Livers of this type were found in eight children while intermediate moderately severe grades of fatty liver were found in six. Where fatty change was slight or moderate the process was usually but not invariably more marked peripherally and this distribution did not appear altered by the presence of infection. In two children the fat vacuoles were either so small or scanty that the sections did not justify the diagnosis of fatty liver and in a third fatty change appeared completely lacking.

Free iron pigment was not seen in more than trifling amounts in any liver section.

Small numbers of bile plugs were seen in 10 cases, and in one child several cells in each lobule were filled with bile. This particular child was jaundiced terminally with a serum bilirubin level of $1.9 \mathrm{mg} .{ }^{\circ}$ and disturbed liver function tests.

Increased cellularity of portal tracts was noted in seven livers and was pronounced in three livers showing focal necrosis. Necrosis was seen in four livers, three of them with severe grades of fatty change. In two children the focal necroses were small and scanty but in the other two the process was more widespread and involved occasionally as much as half a liver lobule in a single necrotic focus. In all four livers the necrosis appeared very recent and was represented chiefly by granular cytoplasmic and nuclear debris among large pools of fat. In one case regenerative activity was suggested by multinucleate cells on the edges of the portal tracts. In none was it possible to demonstrate organisms or parasites. In one other very fatty liver large pools of fat had formed in many liver lobules and resembled the fat cysts described by Hartroft (1950), though they were larger and there were more of them than in his series.

The changes of serous hepatosis (Hill, 1951) were completely absent from these livers.

Silver impregnation methods showed loss of bridging reticulum fibres in fatty livers. This accentuated the length of portal tracts, which happened to be cut longitudinally, but a convincing monolobular fibrosis was never seen and portal cirrhosis was certainly absent. In two livers the portal tracts were unusually bulky in reticulum preparations and short thick processes entered the parenchyma from the portal triads while there was an excessive quantity of collagen in the walls of some central veins. These abnormalities of reticulum pattern were not seen diffusely throughout either of the two sections but only in subcapsular areas and were interpreted as secondary to focal subcapsular atrophy rather than as true fibrosis. Minor variations in the number, thickness and situation of the reticulum fibres were commonly seen but could be found in control material. In the four livers with focal necrosis, changes in reticulum pattern, apart from those secondary to fatty change, were uncommon and slight in degree while the same was true of the liver with the large pools of fat unassociated with cell necrosis. 
Significant histological changes were commonly seen in the pancreas though alterations in the naked-eye appearances were not recorded. Microscopical sections were available from 34 children, and in 28 of them there was atrophy of the acinar cells though not of the islet tissue. In the severest grades of atrophy the cells were represented virtually by bare nuclei alone and this was seen in 17 children. In two of these 17 children the condensation of existing connective tissue was pronounced, but in only one child was there a true fibrosis and here, unlike in all the others, duct obstruction and inflammation were seen though evidence of fibrocystic disease of the pancreas was

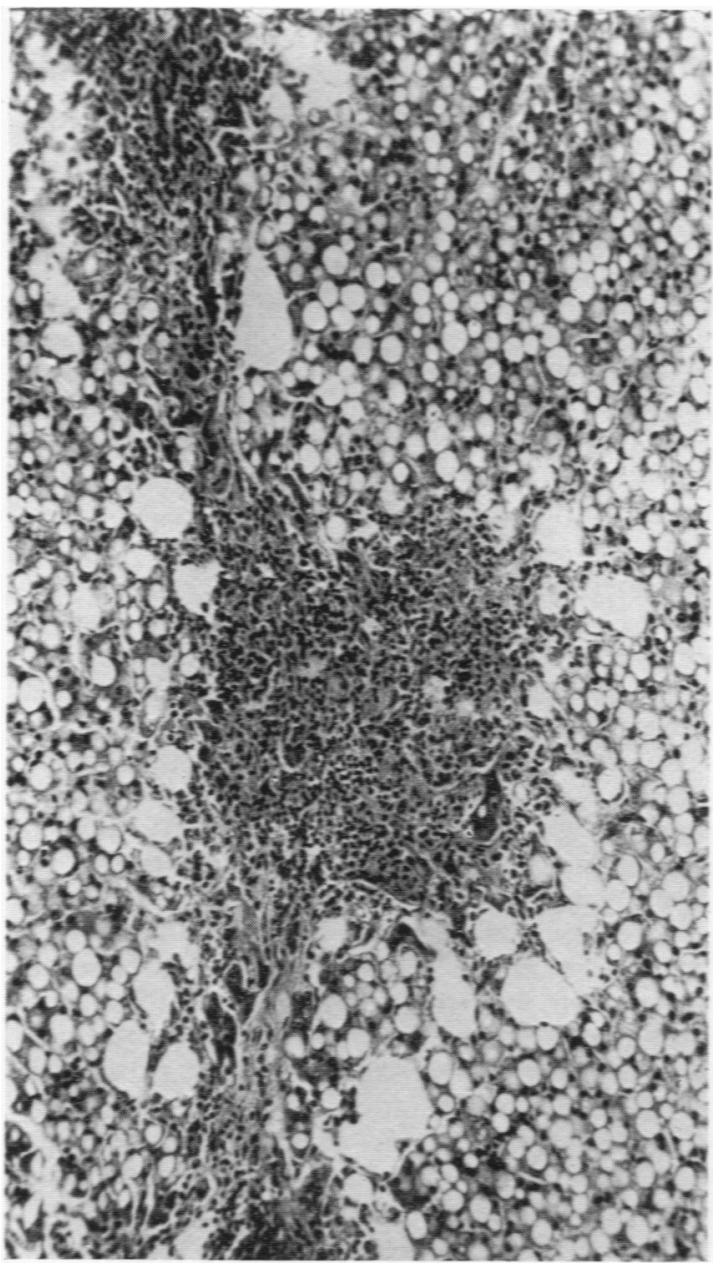

FIG. 1.-Liver from a coloured boy of $2 \frac{1}{2}$ years, showing a severe degree of fatty change. This is accompanied by overcellularity of portal tracts, necrosis of isolated liver cells, attempts at liver cell regeneration and the formation of large pools of fat just beyond the portal tracts. Haematoxylin and eosin $\times 95$. lacking. On histological grounds it seemed clear that the fibrosis was not secondary to simple atrophy with collapse of the pre-existing connective tissue framework of the gland. It was not, however, possible to infer that the pancreatic changes had preceded those in the liver in this particular child. One further microscopical change noted in the pancreas in nine children was the presence of pale pink-staining material in somewhat distended acini. This change corresponds to what Bodian (1952) describes as pancreatosis and what Emery (1951) found in many normal infant pancreases.

The commonest finding in the bones of these children was depressed endochondral ossification,

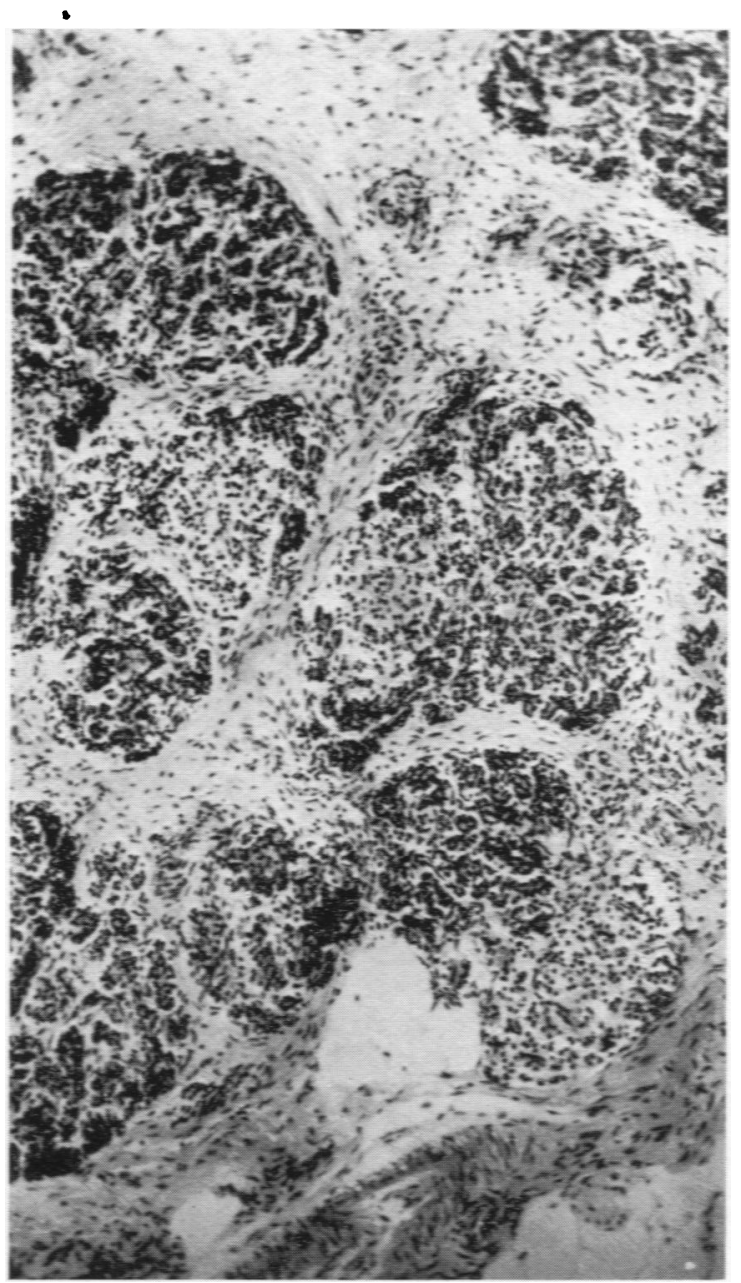

Fig. 2.-Pancreas from the same case in which can be seen atrophy of acinar cells, persistence of islet tissue and pancreatic fibrosis. Haematoxylin and eosin 995 . 
and microscopical evidence of this was obtained in eight of the 12 children from whom sections were available. Swollen costochondral junctions were noted at necropsy in two children but changes compatible with rickets were present in only one of these. In the other there was abnormal vascularization of a bulky zone of hypertrophic cartilage cells and small foci of woven bone formation within this zone. A thin subperiosteal haemorrhage was present in the one other case but the histological features were otherwise normal and the clinical data did not suggest scurvy. Gelatinous degeneration of bone marrow was seen in two cases.

Skeletal muscle was available for study in 15 cases. Abnormal features were atrophy of muscle fibres in six and sarcolemmal nuclear proliferation in five cases. Diaphragmatic muscle was available in eight of these 15 children and in four sarcolemmal nuclear proliferation was again present. Lingual muscle was studied in 17 cases and the same nuclear changes were seen in five of them. Although the body muscles doubtless became depleted of protein in malnutrition one could never be sure how much of the microscopical picture was produced by the atrophy of disuse.

No significant changes were observed in the heart, endocrine organs or lymphoid tissue apart from accidental thymic involution in six cases where the thymus was described. Failure of foetal adrenal cortex to involute was not noted in any child.

One group of important changes remains to be described and will be considered under the heading of infections. In 31 of the 40 children the lungs showed microscopical evidence of pyaemic abscess or bronchopneumonia with or without abscess and pleurisy. Bacteriological examination was done on four occasions, and the organisms isolated were staphylococcus, pneumococcus, coliforms and proteus. Despite the frequency with which gastrointestinal disturbances were recorded in the clinical histories $(85 \%)$, naked-eye lesions in the bowel were uncommon and ulceration was seen only twice. Faeces was cultured from seven children at necropsy, Salmonella group E was isolated from two and no pathogens were found in the other five. Pyaemic abscesses were found in the kidneys of three children at necropsy and in three others the features were those of pyelonephritis. Kidneys not affected by inflammation were sometimes described as pale and a few as having a yellow tinge, the latter contributed presumably by lipoid since fat was seen in the convoluted tubules in frozen sections from three children. Glomeruli in various stages of hyalinization were often seen in this series of children but their number and situation were not different from what is regarded as a natural phenomenon at this age (Morison, 1952).

\section{Discussion}

When the pathological features of malnutrition in Cape Town are compared with those reported from other centres two differences are apparent. The first is the absence of serous hepatosis, which appears to be a distinct and separate entity. The second is the frequency and importance of infections in fatal cases of malnutrition in Cape Town. Necropsy experience appears to indicate quite clearly that infections precipitate the malnourished child into serious illness, determine the admission to hospital and are largely responsible for the high mortality in this condition. It is surely significant that in this series of $\mathbf{4 0}$ children there were only seven in whom an adequate cause of death could not be found, and in some of these, though the clinical history suggested an infection, the necessary bacteriological investigations were not available. This frequency of infections is not unique in Cape Town (Waterlow, 1948; Véghelyi, 1950; Trowell et al., 1954) but nowhere has this feature been admitted as an essential part of malnutrition as seen in the hospital or the necropsy room.

Those aspects of malnutrition which have received greater attention are fatty liver and pancreatic atrophy. Fatty change in the liver is now well established as an accompaniment of infantile malnutrition but the functional significance of this change appears far from settled. Altmann (1948) believed that the evidence was insufficient to prove that fatty change seriously interfered with the liver's functions. Waterlow (1948) on the other hand, on the basis of abnormal bromsulphthalein retention and in the face of serum bilirubin levels not in excess of $1.8 \mathrm{mg}$. \% believed that children with fatty liver disease died from hepatic failure. The diagnosis of hepatic failure, however, should be reserved for those cases which show microscopical evidence of widespread liver necrosis, and it was therefore withheld in the one case in this series where jaundice was present. There is clearly no justification for regarding the focal necroses seen in a few of the livers in this series as evidence of hepatic failure.

Examination of the available material produced no evidence that cirrhosis develops during the course of fatal malnutrition at this age nor were there indications that any focal necrosis would be followed by fibrosis, though frequently repeated lesions of this type might presumably have more serious consequences. Reticulum preparations, on the other hand, showed that appearances resembling 
fibrosis could be produced by subcapsular atrophy, and since liver biopsy specimens often contain tissue from subcapsular areas, ignorance of this possibility might prove misleading.

So far as pancreatic atrophy is concerned, this paper fully confirms the views of Véghelyi (1950), Waterlow (1948) and Davies (1948) that acinar atrophy is a common accompaniment of malnutrition, but the pathological findings do not indicate how this lesion may be linked with the mode of death. There is, moreover, evidence from analysis of duodenal and intestinal secretions that proteolytic ferments at least are still produced by the pancreas in malnutrition (Altmann, 1953). No child in this series showed evidence that simple atrophy of acinar tissue leads to pancreatic fibrosis, while Emery's study of the normal infant's pancreas indicates that considerable caution is required before venturing the diagnosis of pancreatic fibrosis in infants.

Although this investigation cannot add or detract materially from the views held at present on the parts played by the liver and pancreatic lesions in malnutrition, it has shown that infections are of considerable importance in this condition. No one would claim that infections are a cause of malnutrition, but they do in Cape Town appear to be the most important single factor responsible for turning a badly nourished child, not obviously ill, into one so ill that careful hospital treatment is necessary if a fatal outcome is to be avoided.

One last finding needing explanation is the virtual absence of fatty change in the livers of two children and its complete absence in a third. This feature has been encountered by others and explained as the result of treatment, very prolonged periods of malnutrition, the occurrence of both malnutrition and undernutrition in the same child or as a feature of the earliest stages of malnutrition (Waterlow, 1948; Trowell et al., 1954). One or more of the causes enumerated was found to apply in each of the three cases in this series that showed minimal amounts of fat in the liver.

\section{Summary}

The morbid anatomical findings in malnutrition in infants in Cape Town were found to be basically similar to those reported from other parts of the world, except that the condition of serous hepatosis was not seen.

Infections were thought to be an important precipitating factor in causing serious illness in malnourished children and the major factor in determining death.

I wish to acknowledge the help and encouragement given by Professor J. G. Thomson in the preparation of this paper.

\footnotetext{
REFERENCES

Altmann, A. (1948). Clin. Proc., 7, 32.

Bodian, M. (1952). Fibrocystic Disease of the Pancreas. p. 103. London.

Davies, J. N. P. (1948). Lancet, 1, 317

Emery, J. L. (1951). J. Anat. (Lond.), 85, 159.

Gillman. J. and Gilbert, C. (1954). Ann. N.Y. Acad. Sci.. 57, art. 6. p. 737 .

and Gillman, T. (1951). Perspectives in Human Malnutrition. New York.

Hartroft, W. S. (1950). Anat. Rec., 106, 61.

Hill, K. R. (1951). Trans. 10th Conference on Liver Injury, p. 263. Josiah Macy Jr. Foundation. New York.

Morison, J. E. (1952). Foetal and Neonatal Pathology, p. 322.

London. Kwashiorkor. London.

Véghelyi, P. V. (1950). Ann. Pediat. (Basel), 175, 349.

Walters, J. H. and Waterlow, J. C. (1954). Spec. Rep. Ser. med. Res. Coun. (Lond.). No. 285.

Waterlow, J. C. (1948). Ibid., No. 263.
} 